\title{
Circular motion and Polish Doughnuts in NUT spacetime
}

\author{
Paul I. Jefremov \\ ZARM - Centre for Applied Space Technology and Microgravity, University of Bremen, \\ Am Fallturm, 28359 Bremen, Germany \\ email: paul.jefremow@zarm. uni-bremen.de
}

\begin{abstract}
The astrophysical relevance of the NUT spacetime(s) is a matter of debate due to pathological properties exhibited by this solution. However, if it is realised in nature, then we should look for the characteristic imprints of it on possible observations. One of the major sources of data on black hole astrophysics is the accretion process. Using a simple but fully analytical "Polish Doughnuts" model of accretion disk one gets both qualitative and quantitative differences from the Kerr spacetime produced by the presence of the gravitomagnetic charge. The present paper is based on our work Jefremov \& Perlick (2016).
\end{abstract}

Keywords. Accretion disks, hydrodynamics, relativity, black hole physics

\section{Introduction}

Taub-NUT metric is a solution of Einstein field equations [see e. g. Griffiths \& Podolský (2009)] describing a source possessing in addition to the "gravitoelectric charge" (usual mass, $M)$ also the "gravitomagnetic monopole" charge, $n$. In spherical coordinates $(t, r, \theta, \phi)$ the NUT metric reads

$$
g_{\mu \nu} d x^{\mu} d x^{\nu}=\frac{\Delta}{\Sigma}(\mathrm{d} t-2 n(\cos \theta+C) \mathrm{d} \phi)^{2}-\frac{\Sigma}{\Delta} \mathrm{d} r^{2}-\Sigma\left(\mathrm{d} \theta^{2}+\sin ^{2} \theta \mathrm{d} \phi^{2}\right)
$$

where $\Delta=r^{2}-2 M r-n^{2}$ and $\Sigma=r^{2}+n^{2}$. In this expression $C$ is the Manko-Ruiz constant introduced by Manko \& Ruiz (2005), the physical identification of which is not clear. Geodesics in the NUT space-time were studied by Kagramanova et al. (2010). Using their results we derived parameters of circular geodesics and in particular the surface where they are situated (see Jefremov \& Perlick (2016) and the dashed black line on Fig. 1). The location of the circular geodesic orbits is important for the Polish Doughnuts accretion model considered in the next section, since the centre of the configuration moves on a geodesic.

\section{Polish Doughnuts in NUT Spacetime}

The Polish Doughnuts model of matter with constant specific angular momentum rotating around a black hole is remarkable for its fully analytical expression for the density distribution in the resulting toroidal configuration if one assumes the polytropic relation $\left(p=K \rho^{\Gamma}\right)$ for the flow [see e. g. Rezzolla \& Zanotti (2013)]:

$$
\rho(r, \theta)=\left[\frac{\Gamma-1}{K \Gamma}\left[\exp \left(W\left(r_{i n}, \theta_{i n}\right)-W(r, \theta)\right)-1\right]\right]^{1 /(\Gamma-1)},
$$

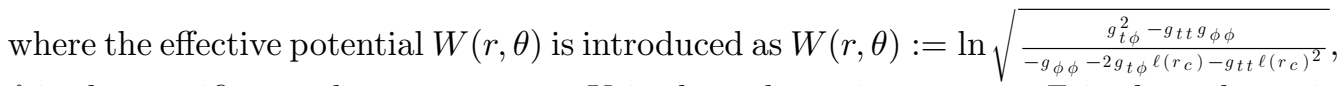
$\ell$ is the specific angular momentum, $K$ is the polytropic constant, $\Gamma$ is the polytropic 

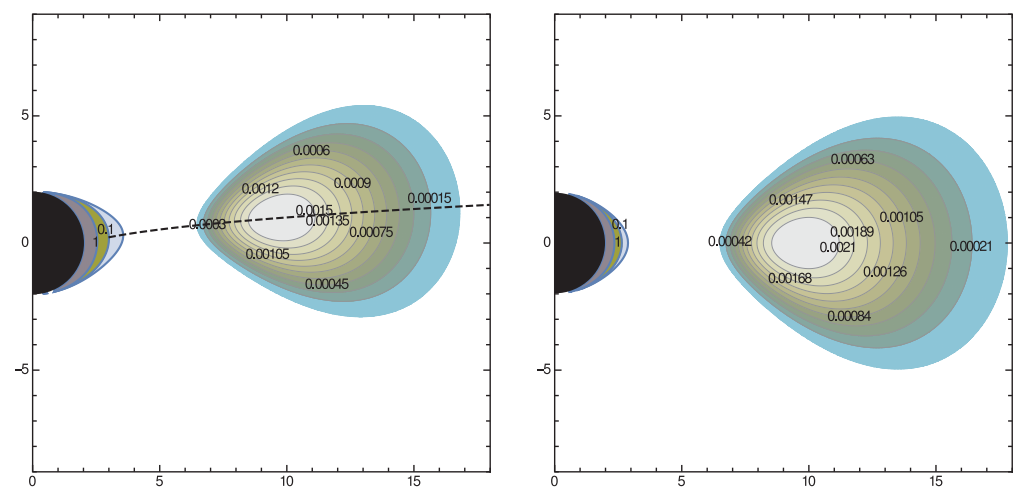

Figure 1. Polish Doughnut with $r_{\text {cen }}=9.7 M, r_{\text {in }}=6.5 M$ and $\Gamma=5 / 3$ and $K=0.2$ in NUT space-time with $n=0.2 M$ (left) and in Kerr space-time with $a=0.2 M$ (right). $M$ is the unit on the axes. Different colours correspond to different values of the matter density $\rho$ in units $\left[1 / M^{2}\right]$. The parameters $r_{\text {cen }}, r_{\text {in }}, \Gamma, K$ are chosen to satisfy the absence of self-gravity and special relativistic effects conditions (Jefremov \& Perlick (2016)). The dashed line on the left picture denotes the surface of circular orbits for the given value of $n$.

\begin{tabular}{|c|c|c|c|c|c|}
\hline \multicolumn{6}{|c|}{\begin{tabular}{|l|l|l|}
$\frac{n}{M}\left|\frac{a}{M}\right| m /\left(10^{-2} M\right)\left|\rho_{\text {cen }} /\left(10^{-4} M^{-2}\right)\right| T_{\text {cen }} /\left(10^{9} \mathrm{~K}\right)$ \\
\end{tabular}} \\
\hline $\mid \begin{array}{lll}0 & 0 & 0\end{array}$ & 5.71 & I & 2.05 & 1 & 7.46 \\
\hline \begin{tabular}{l|l|l|}
0.2 & 0 & \\
\end{tabular} & 5.28 & 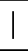 & 1.99 & 1 & 7.01 \\
\hline \begin{tabular}{|l|l|}
0.4 & 0
\end{tabular} & 7.74 & 1 & 1.81 & 1 & 7.60 \\
\hline \begin{tabular}{|l|l|}
0 & 0.2
\end{tabular} & 7.91 & | & 2.52 & I & 8.74 \\
\hline
\end{tabular}

Table 1. Torus mass $m$, density in the centre $\rho_{\text {cen }}$ and temperature in the centre $T_{\text {cen }}$ for different values of NUT and Kerr parameters with the values of model parameters as in Fig. 1. The values for $n, a$ (Kerr parameter), $m, \rho_{\text {cen }}$ are given in terms of the black-hole mass $M$, the temperature is given in Kelvins.

exponent and $r_{i n}$ (inner edge) and $r_{c}$ (centre) are parameters of the configuration. Choosing values of these parameters one obtains the density distribution in the torus (Fig. 1) and assuming further the ideal gas equation of state, the temperature and other characteristics of it. One clearly sees the qualitative difference in the shape of the torus which has its centre outside of the equatorial plane, where it lies in Kerr spacetime, and quantitative dependence of the physical properties of the torus on $n$ (see Table 1).

\section{Conclusions}

NUT spacetime exhibits qualitatively different properties compared to the Kerr spacetime in the considered model of the accretion disc. The presence of the NUT parameter makes the configuration less massive and less hot in the centre than in the Kerr or Schwarzschild case.

\section{References}

Jefremov, P. I. \& Perlick, V. 2016, arXiv:1608.06218, submitted to Class. Quant. Grav. Griffiths, J. B. \& Podolský, J., Exact Space-Times in Einstein's General Relativity, CUP (2009). Manko, V. S. \& Ruiz, E. 2005, Class. Quantum Grav., 22, 3555.

Kagramanova, V., Kunz, J., Hackmann, E., \& Lämmerzahl, C. 2010, Phys. Rev. D, 81, 124044. Rezzolla, L. \& Zanotti, O., Relativistic Hydrodynamics, OUP, 2013. 